\title{
Criminologie
}

\section{Éditorial : la nouvelle formule de Criminologie}

\section{Pierre Robert}

Volume 22, numéro 2, 1989

Le milieu criminel

URI : https://id.erudit.org/iderudit/017278ar

DOI : https://doi.org/10.7202/017278ar

Aller au sommaire du numéro

Éditeur(s)

Les Presses de l'Université de Montréal

ISSN

0316-0041 (imprimé)

1492-1367 (numérique)

Découvrir la revue

Citer ce document

Robert, P. (1989). Éditorial : la nouvelle formule de Criminologie. Criminologie, 22(2), 3-4. https://doi.org/10.7202/017278ar d'utilisation que vous pouvez consulter en ligne.

https://apropos.erudit.org/fr/usagers/politique-dutilisation/ 
Depuis sa fondation par Denis Szabo en 1968, la revue Criminologie n'a cessé d'évoluer et de s'adapter aux exigences d'une diffusion de qualité des connaissances et de la réflexion criminologiques. Revue de transfert d'information scientifique basée sur des résultats de recherche, la revue Criminologie s'était renouvelée une première fois, en 1975, en devenant thématique. Le huitième volume de la revue était alors entièrement consacré à la délinquance juvénile au Québec. La revue qui, en cette occasion, avait substitué son nom actuel à sa première dénomination d'Acta Criminologica, s'engageait résolument dans la présentation prioritaire des travaux de la communauté intellectuelle québécoise.

En 1977, sa dixième année de publication, Criminologie devenait semestrielle. Néanmoins, la revue conservait sa vocation thématique. Le volume $\mathrm{X}$ de la revue proposait ainsi un premier numéro sur la criminalité des affaires et un deuxième, à l'automne, sur la criminologie au Québec.

Jusqu'à maintenant, chaque numéro traitait ainsi d'un thème unique. Toutefois, cette caractéristique, parce qu'elle s'appliquait à tous les articles d'une livraison, s'est avérée une contrainte, parfois même une véritable entrave au plan des politiques rédactionnelles. En effet, l'exclusivité du thème dissuadait d'éventuels collaborateurs à présenter leurs productions de recherche soit parce qu'elles n'étaient pas reliées au thème retenu, soit parce que le projet du numéro thématique pertinent différait considérablement la publication des résultats d'une recherche récente.

Désormais, Criminologie, tout en conservant sa caractéristique de revue thématique, consacre une partie de chaque numéro à la publication d'articles hors-thème ou reliés à un sujet d'actualité.

Les articles thématiques sont maintenant regroupés dans un dossier de trois ou quatre articles. En principe, il seront présentés dans le cadre de l'éditorial. Exceptionnellement, dans la présente livraison, le dossier consacré au milieu criminel fait l'objet d'une présentation sous forme de plaidoyer, sinon de plaidoirie, par Pierre Tremblay, en faveur de la sociologie criminelle. C'est que la revue expérimente également,

*Professeur au Département des sciences juridiques de l'Université du Québec à Montréal, case postale 8888, succursale A, Montréal, Québec H3C 3P8. 
dans ce numéro, en présentant un dossier thématique consacré non pas à un sujet ou à un phénomène particulier de criminalité mais plutôt à une approche et une problématique reliées à l'analyse du milieu criminel. La revue Criminologie soumet donc à l'appréciation de ses lecteurs cette formule inédite par laquelle une équipe de chercheurs livre ses réflexions et les résulats de ses travaux.

Les deux derniers articles de ce numéro sont consacrés à la géographie criminelle et à l'histoire du contrôle social. Tout d'abord, Maurice Cusson propose une synthèse de la tradition de la géographie ou de l'écologie criminelle de Montréal dans un article intitulé «Zones urbaines criminelles». Il s'agit d'une analyse de la littérature sur la formation et les caractéristiques des aires géographiques qui révèlent un indice élevé de criminalité. Cet article de Maurice Cusson développe également une réflexion sur la sociologie criminelle qui n'est pas sans rapport avec le dossier thématique sur le milieu criminel. Puis JeanFrançois Leclerc présente une synthèse de l'histoire de la Sûreté du Québec. S'inspirant de l'histoire des institutions, l'auteur y analyse l'évolution administrative et juridique de ce corps policier révélant ainsi un aspect rasement traité des institutions de la justice pénale. 